\title{
Point-of-care lung ultrasound for diagnosis of Pneumocystis jirovecii pneumonia: notes from the field
}

\author{
Maria Teresa Giordani ${ }^{1 *}$ DD, Francesca Tamarozzi ${ }^{2}$, Daniel Kaminstein ${ }^{3}$, Enrico Brunetti ${ }^{4}$ and Tom Heller ${ }^{5}$
}

\begin{abstract}
Background: Thoracic ultrasound is helpful to evaluate lung pathology in patients with acute dyspnea. Several studies have demonstrated the efficacy of point-of-care ultrasound in patients with extrapulmonary TB and HIV coinfection. This retrospective, open-label case-control study explores the role of lung ultrasound in the diagnosis of Pneumocystis jirovecii pneumonia (PJP) in HIV-positive patients. In particular, it highlights the potential role of specific sonographic features that may be unique to this population.

Methods: The record of all HIV-positive patients admitted from 1.1.2013 to 31.6.2017 to the Department of Infectious Diseases and Tropical Medicine of san Bortolo Hospital, Vicenza, Italy, with a discharge diagnosis of acute lung injury (ALI) and who received point-of-care ultrasound of the chest for clinical purposes was included in the analysis. The patients were scanned according with the evidence-based recommendation.

Results: Of 273 HIV-positive patients whose records were reviewed, 81 (29.6\%) were diagnosed with ALI. Complete documentation was available for 24 patients, of which 14 (58.3\%) had microbiologically confirmed PJP (PJP+) and 10 (41.7\%) had other conditions (PJP-). B-lines, subpleural consolidations, and cystic changes were significantly more frequent in patients with PJP (14/14 vs. 6/10, $p=0.0198 ; 14 / 14$ vs. 4/10, $p=0.0016 ; 8 / 14$ vs. $0 / 10, p=0.0019$, respectively). In particular, B-lines and subpleural consolidations were present in all PJP + patients in our cohort giving a sensitivity of 100\%, but their specificity was low (45 and 60\%, respectively). On the contrary, the presence of consolidations with cystic changes had a very high specificity for PJP (100\%), but low sensitivity (57\%). Pleural effusions and consolidations with linear air bronchograms were not observed in PJP+ patients.

Conclusions: B-lines, subpleural consolidations, and cystic changes are suggestive of PJP. Lung consolidation with air bronchograms and pleural effusion should prompt suspicion of other etiologies. These findings have the potential to be useful in the daily management of HIV-positive patients in resource-limited settings where other diagnostic tools are rarely available.
\end{abstract}

Keywords: POC ultrasound, Lung, Pneumocystis pneumonia, AIDS, HIV, Pneumonia, Lung ultrasound

\section{Background}

The interpretation of artifacts created during ultrasound of the thoracic cavity was first described over 20 years ago [1]. Since then thoracic ultrasound has become a well-established imaging modality used by clinicians at the bedside of acutely dyspneic patient. The BLUE

\footnotetext{
*Correspondence: giordanimt@gmail.com

1 Infectious and Tropical Diseases Unit, San Bortolo Hospital, Via Rodolfi 37,36100 Vicenza, Italy

Full list of author information is available at the end of the article
}

protocol [2] is a helpful approach to lung ultrasound as it outlines a step-by-step decision tree to differentiate between potential causes of dyspnea. In low-resource settings where even a basic chest X-ray (CXR) may not be available [3] ultrasound of the chest has profound implications. Point-of-care ultrasound (POCUS) has an established role as a diagnostic tool in HIV-positive patients with extrapulmonary tuberculosis (TB) [4], and has been shown to complement CXR [5]. Furthermore, it is a frequent POCUS application in settings with high TB and 
HIV prevalence [6]. Recent case series describe sonographic lung finding seen in HIV-positive patients [7] and in patients with miliary TB [8].

Pneumocystis jirovecii pneumonia (PJP) is one of the most common opportunistic infections in HIV-positive patients [9] who are not receiving prophylaxis especially when CD4- counts fall below 200 cells $/ \mathrm{mm}^{3}$ [10]. Without timely diagnosis and treatment PJP carries a high mortality [11], though survival rates have increased in the era of antiretroviral therapy (ART) [12]. Diagnosis of PJP poses a significant challenge, due to its non-specific clinical presentation. Computed tomography (CT) of the chest remains the mainstay of diagnosis [13]. In cross-sectional CT imaging, PJP presents with bilateral geographic or patchy ground-glass opacities with septal thickening, lung consolidation, nodules, cysts, and pneumothorax, while pleural effusions are uncommon [14]. Microbiological confirmation relies on bronchoalveolar lavage (BAL) followed by microscopy or PCR. BAL is invasive, poorly tolerated by patients with respiratory distress and analysis requires a well-equipped laboratory with specific expertise.

In resource-limited settings, where the majority of HIV-positive patients live, the risk of PJP is high [15]; CT and bronchoscopy are generally not available. As POCUS has been found a promising tool for other diseases in these settings $[16,17]$, our study aimed to explore the diagnostic potential of sonographic lung features in the diagnosis of HIV-positive patients with PJP.

\section{Methods}

\section{Study design and data collection}

This retrospective unmatched case-control study of HIV-positive patients with and without microbiologically confirmed PJP was conducted in the Tropical Medicine and Infectious Diseases Department, San Bortolo Hospital, Vicenza, Italy, that cares for approximately 1000 HIVpositive individuals yearly.

The department operates a clinical ultrasound service including bedside POCUS. Chest POCUS is performed by an infectious disease specialist (MTG) using an Aplio XG Model SSA-790A with a $3.5-\mathrm{MHz}$ convex and an 8-MHz linear probe (Toshiba, Tokyo, Japan). All patients included in this study were examined with both probes; the anterior, posterior, and lateral chest wall was bilaterally scanned in longitudinal (cranio-caudal orientation) and transverse (aligned with the intercostal space) orientation from apex to base with patients in supine, sitting or near-to-supine position, trying to achieve the maximum extension of the visible pleural line. No standard protocol was followed, although the International evidence-based recommendations for point-of-care lung ultrasound were taken into consideration [18].
All records of HIV-positive patients admitted from 1.1.2013 to 31.6.2017 were reviewed and patients with a discharge diagnosis of acute lung injury (ALI) according to Diagnosis-Related-Group (DRG) classification (http:// www.salute.gov.it/imgs/C.pdf) were identified. Inclusion criteria were as follows: (1) HIV infection; (2) discharge diagnosis of ALI; (3) BAL followed by microbiological examination for $P$. jirovecii (microscopy or PCR); and (4) POCUS of the chest performed during the first week of admission blinded to CT scan findings. Extracted patient information included demographics, history of ART, CD4+ count, HIV viral load, microbiological, and imaging data. Stored static images and video files of all patients were reviewed for the study (Additional files 1, $2,3)$.

\section{Statistical methods}

Patients included were classified as microbiologically confirmed PJP (PJP+) or control patients with ALI without microbiologically confirmed PJP (PJP-). Data are presented as medians, interquartile ranges (IQR), and counts with percentages, as appropriate. Differences in proportions were analyzed by Fisher's Exact test; continuous variables by Mann-Whitney- $U$ test. All tests were two-sided; $p$-values $<0.05$ were considered significant. Analyses were done using MedCalc version 17.8 (Ostend, Belgium).

\section{Description of ultrasound findings}

An ultrasound was considered normal when a normal lung surface, a pleural line with lung sliding, and A-lines were present and a maximum of two B-lines per single view was seen. Pathological ultrasound findings extracted for analysis were increased number of B-lines, pleural effusions, subpleural consolidation, lung consolidation with air bronchogram patterns or a cystic pattern, and presence of pneumothorax. Ultrasound findings are described in detail in Table 1.

\section{Results \\ Patient characteristics}

The records of 273 HIV-positive patients admitted during the study period were reviewed. 81 patients (29.6\%) were diagnosed with ALI. Complete documentation was available for 24 patients, 18 males, and 6 females, of which 14 (58.3\%) had microbiologically confirmed PJP $(\mathrm{PJP}+)$ and $10(41.7 \%)$ were diagnosed with other conditions (PJP-). Clinical and demographic data are summarized in Table 2.

The frequency of lung ultrasound findings is summarized in Table 3. B-lines, subpleural consolidations, and cystic changes were more frequently seen in patients with PJP, and reached statistical significance. In particular, 
Table 1 Clinical and demographic characteristics of the patients included in the study

\begin{tabular}{|c|c|c|c|c|}
\hline & Total $n=24(100 \%)$ & $\mathrm{PJP}+n=14(58.33 \%)$ & $\mathrm{PJP}-n=10(41.66 \%)$ & $p$-value \\
\hline Sex males/females (\% males) & $18 / 6(75.00 \%)$ & $13 / 1(92.85 \%)$ & $5 / 5(50.00 \%)$ & 0.0501 \\
\hline Median age in years (IQR) & $44(36-53)$ & $43(35-54)$ & $44(41-51)$ & 0.8602 \\
\hline \multicolumn{5}{|l|}{ Ethnic origin $(n)$} \\
\hline Caucasian & 18 & 11 & 7 & \\
\hline African & 3 & 1 & 2 & \\
\hline Latin-American & 3 & 2 & 1 & \\
\hline 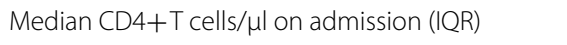 & $56.5(18-172.25)$ & $39.5(16-73.5)$ & $137.5(59.75-325)$ & 0.0608 \\
\hline Median viral load in copies/ml on admission (IQR) & $345,000(91,427-607,400)$ & $262,450(61,291-587,150)$ & $391,200(195,400-790,400)$ & 0.8291 \\
\hline PJP as first AIDS-defining condition & n.a. & 13/14 (92.85\%) & n.a. & \\
\hline Final diagnosis & - & PJP & See note ${ }^{a}$ & \\
\hline
\end{tabular}

PJP, Pneumocystis jirovecii pneumonia; IQR, inter quartile range; n.a., not applicable

a Final diagnoses of PJP-patients: bacterial pneumonia $(n=3), T B(n=3)$, disseminated Cytomegalovirus infection $(n=1)$ and Mycobacterium avium complex infection $(n=1)$ with lung involvement, non-Hodgkin's T cell lymphoma $(n=1)$ and adenocarcinoma $(n=1)$ of the lung

Table 2 Diagnostic performances of the lung ultrasound findings

\begin{tabular}{|c|c|c|c|c|c|c|c|c|c|}
\hline Lung ultrasound sign & $\begin{array}{l}\text { PJP+ } \\
n(\%)\end{array}$ & $\begin{array}{l}\text { PJP- } \\
n(\%)\end{array}$ & $p$-value & $\begin{array}{l}\text { Se } \\
(95 \% \mathrm{Cl})\end{array}$ & $\begin{array}{l}\text { Sp } \\
(95 \% \mathrm{Cl})\end{array}$ & $\begin{array}{l}\text { PPV } \\
(95 \% \mathrm{Cl})\end{array}$ & $\begin{array}{l}\text { NPV } \\
(95 \% \mathrm{Cl})\end{array}$ & $\begin{array}{l}\text { LR+ } \\
(95 \% \mathrm{Cl})\end{array}$ & $\begin{array}{l}\text { LR- } \\
(95 \% \mathrm{Cl})\end{array}$ \\
\hline $\begin{array}{l}\text { B-lines ( }>2 \text { per view) no } \\
\text { B-lines in the scan }\end{array}$ & $14(100 \%)$ & $\begin{array}{l}6 \\
(60 \%)\end{array}$ & 0.0198 & $\begin{array}{l}100 \% \\
(76.84-100)\end{array}$ & $\begin{array}{l}45.45 \% \\
(16.75-76.62)\end{array}$ & $\begin{array}{l}70 \% \\
(57.64-80.01)\end{array}$ & $\begin{array}{l}100 \% \\
-\end{array}$ & $\begin{array}{l}1.83 \\
(1.07-3.14)\end{array}$ & $\begin{array}{l}0.00 \\
-\end{array}$ \\
\hline Pleural effusion & $\begin{array}{l}0 \\
(0 \%)^{\mathrm{a}}\end{array}$ & $\begin{array}{l}5 \\
(50 \%)\end{array}$ & 0.0059 & $\begin{array}{l}0.00 \% \\
(0.00-23.16)\end{array}$ & $\begin{array}{l}50.00 \% \\
(18.71-81.29)\end{array}$ & $\begin{array}{l}0.00 \% \\
-\end{array}$ & $\begin{array}{l}26.32 \% \\
(16.12-39.90)\end{array}$ & $\begin{array}{l}0.00 \\
-\end{array}$ & $\begin{array}{l}2.00 \\
(1.08-3.27)\end{array}$ \\
\hline Pneumothorax & $\begin{array}{l}4 \\
(28.5 \%)\end{array}$ & $\begin{array}{l}1 \\
(10 \%)\end{array}$ & 0.3577 & $\begin{array}{l}40.00 \% \\
(12.16-73.76)\end{array}$ & $\begin{array}{l}90.00 \% \\
(55.50-99.75)\end{array}$ & $\begin{array}{l}80.00 \% \\
(34.93-96.75)\end{array}$ & $\begin{array}{l}60.00 \% \\
(46.48-72.15)\end{array}$ & $\begin{array}{l}4.00 \\
(0.54-29.81)\end{array}$ & $\begin{array}{l}0.67 \\
(0.39-1.15)\end{array}$ \\
\hline Subpleural consolidation & $14(100 \%)$ & $\begin{array}{l}4 \\
(40 \%)\end{array}$ & 0.0016 & $\begin{array}{l}100 \% \\
(76.84-100)\end{array}$ & $\begin{array}{l}60.00 \% \\
(16.24-87.84)\end{array}$ & $\begin{array}{l}77.78 \% \\
(62.10-88.20)\end{array}$ & $\begin{array}{l}100 \% \\
-\end{array}$ & $\begin{array}{l}2.50 \\
(1.17-5.34)\end{array}$ & $\begin{array}{l}0.00 \\
-\end{array}$ \\
\hline $\begin{array}{l}\text { Lung consolidation, any lung } \\
\text { consolidations with "cystic" } \\
\text { changes at the pulmonary } \\
\text { basis }\end{array}$ & $\begin{array}{l}8 \\
(57.1 \%)\end{array}$ & $\begin{array}{l}5 \\
(50 \%)\end{array}$ & 1.0000 & $\begin{array}{l}57.14 \% \\
(28.86-82.34)\end{array}$ & $\begin{array}{l}50.00 \% \\
(18.71-81.29)\end{array}$ & $\begin{array}{l}61.54 \% \\
(42.60-77.52)\end{array}$ & $\begin{array}{l}45.45 \% \\
(25.95-66.46)\end{array}$ & $\begin{array}{l}1.14 \\
(0.53-2.46)\end{array}$ & $\begin{array}{l}0.86 \\
(0.36-2.04)\end{array}$ \\
\hline $\begin{array}{l}\text { Lung consolidation with air } \\
\text { bronchogram }\end{array}$ & $\begin{array}{l}0 \\
(0 \%)\end{array}$ & $\begin{array}{l}4 \\
(40 \%)\end{array}$ & 0.0198 & $\begin{array}{l}0.00 \% \\
(0.00-23.16)\end{array}$ & $\begin{array}{l}60.00 \% \\
(26.24-87.84)\end{array}$ & $\begin{array}{l}0.00 \% \\
-\end{array}$ & $\begin{array}{l}30.00 \% \\
(20.53-41.55)\end{array}$ & $\begin{array}{l}0.00 \\
-\end{array}$ & $\begin{array}{l}1.67 \\
(1.00-2.76)\end{array}$ \\
\hline $\begin{array}{l}\text { Lung consolidation with cystic } \\
\text { pattern }\end{array}$ & $\begin{array}{l}8 \\
(57.1 \%)\end{array}$ & $\begin{array}{l}0 \\
(0 \%)\end{array}$ & 0.0019 & $\begin{array}{l}57.14 \% \\
(28.86-82.34)\end{array}$ & $\begin{array}{l}100 \% \\
(69.15-100)\end{array}$ & $\begin{array}{l}100 \% \\
-\end{array}$ & $\begin{array}{l}62.50 \\
(47.65-75.32)\end{array}$ & - & $\begin{array}{l}0.43 \\
0.23-0.78\end{array}$ \\
\hline
\end{tabular}

PJP, Pneumocystis jirovecii pneumonia; Se, sensitivity; Sp, specificity; PPV, positive predictive value; NPV, negative predictive value; LR+, positive likelihood ratio; LR-, negative likelihood ratio; $\mathrm{Cl}$, confidence interval

a One patient developed bilateral pleural effusion later during the stay in the Intensive Care Unit

B-lines and subpleural consolidations were present in all PJP+ patients in our cohort giving a sensitivity of $100 \%$. The specificity of these two findings was low (45 and 60\%, respectively). The presence of consolidations with cystic changes had a very high specificity for PJP. Pleural effusions and consolidations with linear air bronchograms were not seen in PJP+ patients.

As most HIV-positive patients admitted for acute lung disease underwent routine CXR and CT scan, we were able to compare the POCUS findings with radiologic findings from other modalities. Examples from three patients are shown in Table 4. We found a strong correlation between the imaging pattern seen on ultrasound and that of other imaging modalities. Of the six PJP+patients without "cystic changes," three had CT scans demonstrating normal air-filled lung throughout the subpleural region, two had only intralobular opacities, and one patient had physical limitations to the scan (pectus excavatum).

\section{Discussion}

This is to our knowledge the first study systematically reporting POCUS findings of the lung in HIV-related PJP. Ultrasound patterns for several lung diseases in HIVpositive patients have been described in a recently published case series [7]; however, the diagnostic accuracy of 


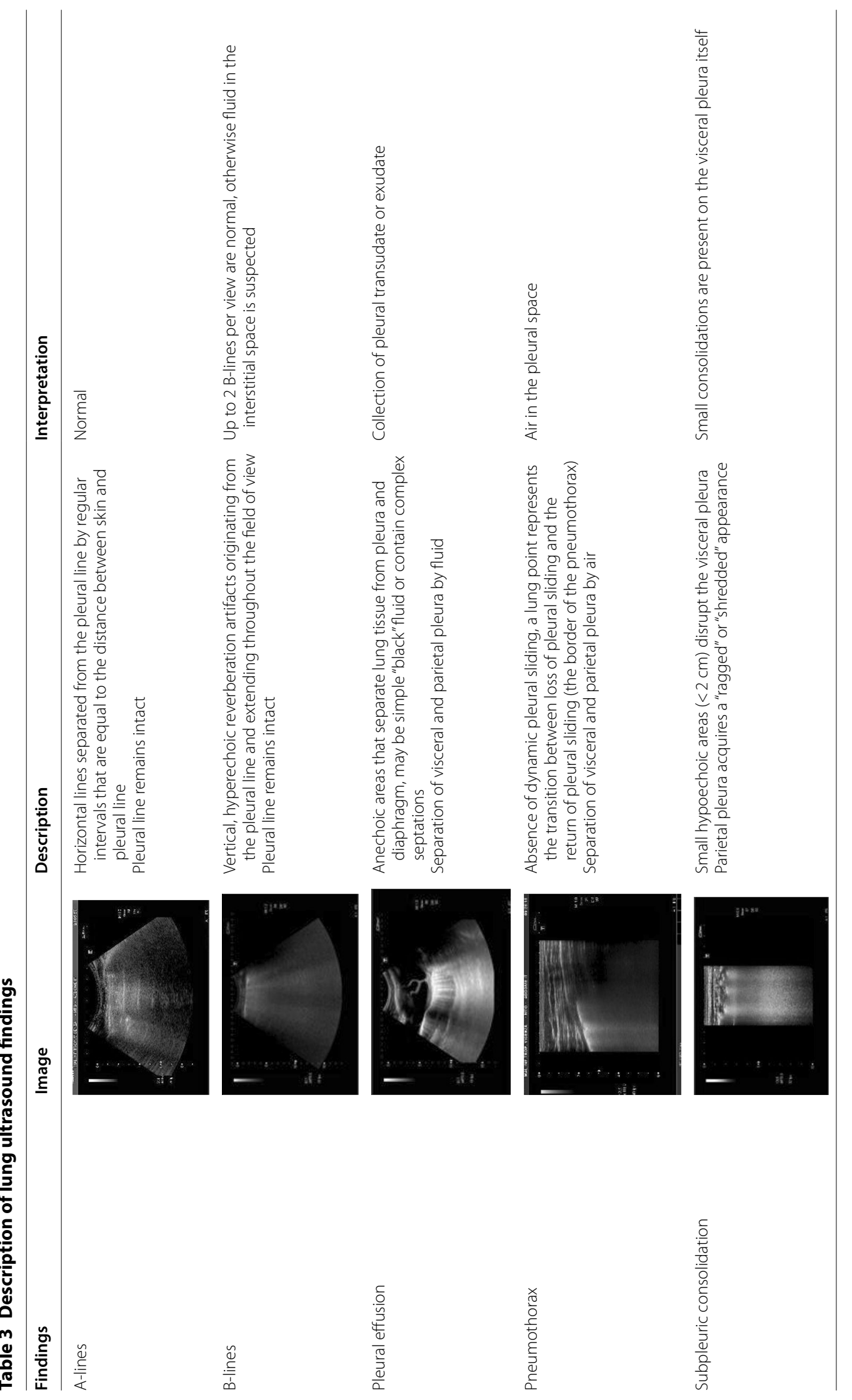




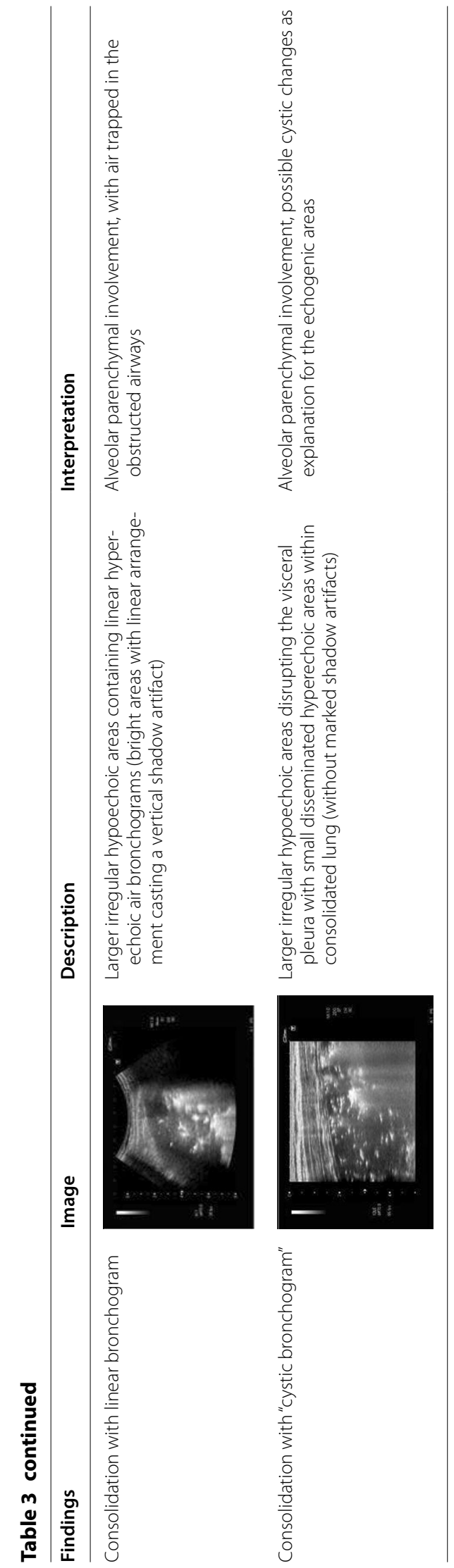


Table 4 Comparison of findings on CT scan, CXR, and ultrasound of three patients with Pneumocystis jirovecii pneumonia PJP

\begin{tabular}{|c|c|c|c|}
\hline & CT scan & CXR & Ultrasound \\
\hline Patient 1 & & & \\
\hline Patient 2 & & & \\
\hline Patient 3 & & & \\
\hline
\end{tabular}

The infection spares the subpleural areas in patient 1 , involves mainly the subpleural areas in patient 2 , and involves wider lung parenchyma in patient 3

chest POCUS in this patient population has never been assessed. Our study suggests that there are thoracic ultrasound findings with significant predictive value for PJP in HIV-positive patients. B-lines, subpleural consolidations and cystic changes are suggestive of PJP. Lung consolidation with air bronchograms and pleural effusion should prompt suspicion of other etiologies.

In particular, the finding of "cystic changes" needs to be highlighted, as it was very specific in our cohort. The sign was characterized by a hypoechoic consolidation including widely scattered echogenic regions suggestive of aircontaining cysts. The pattern was consistently present in serial scans of patients and seems to correlate with the type of lung consolidation seen on CT scan. The typical CT features include a "ground glass" pattern, septal thickening, and walled cysts next to the areas of groundglass attenuation [14]. These may represent pathological changes of lung tissue with filling of the bronchioles and alveoli with foamy debris and macrophages as well as fungal cysts demonstrated in autopsies [18].

Our results should be considered preliminary, given the small numbers and the fact that a single operator performed the ultrasounds. The strength of this study is the use of a referral setting where all diagnostic methods considered to be the gold standard for diagnosing PJP (bronchoscopy, PCR, and CT scan) are routinely available. The discharge diagnosis in our population carries thus a high level of certainty.
Consolidation with "cystic changes," the most specific ultrasound finding, was only detected in $57 \%$ of $\mathrm{PJP}+$ patients and thus has a suboptimal sensitivity. This may be in part due to the inherent limitations in scanning critically ill, dyspneic patients. Also the inhomogeneous spatial distribution of the inflammatory changes may add to this problem. Early stages of the infection seem to spare the subpleural areas [19]. Normal air-filled lung prevents ultrasound from detecting underlying areas of consolidation. Nevertheless, the presence of the pattern seems helpful to "rule-in" the diagnosis.

While no single finding yielded optimal sensitivity and specificity a combination of the described findings on thoracic ultrasound could be helpful in informing clinicians about the likelihood of various lung pathologies in $\mathrm{HIV}+$ patients admitted with ALI.

\section{Conclusion}

POCUS is useful in diagnostic work-up of HIV-positive patients with acute lung disease and ultrasound patterns can help in diagnosing PJP. The use of lung POCUS poses an attractive option for HIV-patients as it combines affordability, non-invasiveness, and availability at patients' bedside regardless of clinical condition. This is even more relevant in resource-limited settings where the majority of patients reside. Our case series should encourage larger prospective studies to validate the 
described thoracic ultrasound findings in HIV-positive patients.

\section{Additional files}

Additional file 1: Clip SA. Subpleural consolidation: a hypoechoic area is visible in the superficial parts of the lung less than $2 \mathrm{~cm}$ below the pleural line.

Additional file 2: Clip SB. Lung consolidation with linear air bronchogram: large hypoechoic area (consolidation) visible containing hyperechoic bright areas casting a vertical shadow artifact. The bright areas are distributed in a linear pattern representing air trapped in the bronchi (sonographic air bronchogram).

Additional file 3: Clip SC. Lung consolidation with cystic changes: large hypoechoic area (consolidation) disrupting the visceral pleura with small, diffusely disseminated hyperechoic areas within the consolidated lung (without marked shadow artifacts). The spatial distribution of is not linear as in clip B.

\section{Abbreviations}

CXR: X-ray; POCUS: point-of-care ultrasound; TB: tuberculosis; PJP: Pneumocystis jirovecii pneumonia; CT: computed tomography; ART: antiretroviral therapy; BAL: bronchoalveolar lavage; ALl: acute lung injury.

\section{Authors' contributions}

MTG conceived the study, performed the ultrasound exams, and collected the data. FT analyzed the data. MTG, FT, DK, and TH drafted the manuscript All authors critically discussed the results and approved the paper. All authors read and approved the final manuscript.

\section{Authors' information}

MTG is a full time clinician in Infectious Diseases and Tropical Medicine in San Bortolo Hospital, Vicenza, Italy. She started using ultrasound, POCUS and interventional US in the setting of Infectious and Tropical Diseases and HIV/ TB patients in 1995 and exported her experience in remote settings with very constrained resources as Tibetan Plateau, Andes, and rural Africa.

FT graduated in medicine from Pavia University (Italy) in 2009, was granted her Ph.D. from the Liverpool School of Tropical Medicine (UK) in 2012 and her specialization in microbiology and virology from Milan University (Italy) in 2017. She is currently working in the Center for Tropical Diseases of Negrar hospital (Verona, Italy). Her broad professional research focus is the diagnosis of parasitic disease and, within this, the use of ultrasound for the diagnosis and epidemiology of parasitic infections.

DK is a full time practicing emergency medicine physician who holds dual fellowship degrees in Global Health and Ultrasound. He received a diploma in tropical medicine from the London School of Hygiene and Tropical Medicine. $\mathrm{He}$ is extensively involved in ultrasound training and research globally and serves as co-director of several ultrasound training courses in Africa and South America. His primary research interest is the application of ultrasound in the evaluation of tropical infectious diseases. He helps lead his university's ultrasound center and is passionate about advancing clinician ultrasound around the globe.

EB is an Infectious Disease clinician with over 30 years of experience in clinical ultrasound, working in a 900-bed teaching hospital in Pavia, Italy. He is a specialist in Internal Medicine and in Infectious Disease and an Associate Professor of Infectious Diseases at the University of Pavia. His research interests include the clinical management of a number of neglected tropical diseases and he has ongoing collaborations in this field with institutions in Europe, Asia, Africa, and the Americas. He directs the international Short Course on Ultrasound in Infectious Diseases and Tropical Medicine, held yearly at the University of Pavia.

TH graduated from medical school at the Technical University Munich, Germany in 1995. He specialized in general internal medicine, as well as in infectious diseases and worked in Germany, Saudi Arabia, and South Africa. He is currently working as a clinical advisor at Lighthouse Trust, operating three large HIV/TB clinic in Malawi. Throughout his career, his research interests were ultrasound applications in the resource-limited setting and sonographic diagnosis and treatment of infectious diseases. He has conducted ultrasound trainings in Germany, Italy, Zimbabwe, Ethiopia, South Africa, Ghana, Malawi, Kuwait, Saudi Arabia, Peru, and Laos.

\section{Author details}

${ }^{1}$ Infectious and Tropical Diseases Unit, San Bortolo Hospital, Via Rodolfi 37, 36100 Vicenza, Italy. ${ }^{2}$ Center for Tropical Diseases, Sacro Cuore-Don Calabria Hospital, Negrar, Verona, Italy. ${ }^{3}$ Department of Emergency Medicine and Hospitalist Service, Medical College of Georgia at Augusta University, Augusta, GA, USA. ${ }^{4}$ Division of Infectious and Tropical Diseases, University of Pavia, IRCCS S. Matteo Hospital Foundation, Pavia, Italy. ${ }^{5}$ Lighthouse Clinic, Lilongwe, Malawi.

\section{Acknowledgements}

We thank Sergio Savastano, MD, for reviewing CXR and CT scans. We thank the U.L.SS. 8 Berica Service Management Control Office for the support, and overall Colleagues and nurses of the San Bortolo Hospital, Vicenza, Italy, for having worked as a tight-knit team with these patients.

\section{Competing interests}

The authors declare that they have no competing interests.

\section{Availability of data}

Data about DRGs were directly provided from the san Bortolo Hospital (U.L.SS. 8 Berica Service Management Control, http://www.aulss8.veneto.it/nodo. php/3128).

All material of this study is in possession of the corresponding author and Hospital Service Management Control Office.

\section{Ethics approval and consent to participate}

As this retrospective study analyzed anonymized clinical data obtained from routine procedures no ethics approval nor consent to participate was required.

\section{Funding}

This article received no funding or grants.

\section{Publisher's Note}

Springer Nature remains neutral with regard to jurisdictional claims in published maps and institutional affiliations.

Received: 18 February 2018 Accepted: 9 April 2018

Published online: 17 April 2018

\section{References}

1. Lichtenstein D, Mézière G, Biderman P, Gepner A, Barré O (1997) The comet-tail artifact, an ultrasound sign of alveolar-interstitial syndrome. Am J Respir Crit Care Med 156:1640-1646

2. Lichtenstein DA, Mezière GA (2008) Relevance of lung ultrasound in the diagnosis of acute respiratory failure. Chest 134:117-125

3. Pedrazzoli D, Lalli M, Boccia D, Houben R, Kranzer K (2017) Can tuberculosis patients in resource-constrained settings afford chest radiography? Eur Respir J 49:1601877

4. Heller T, Wallrauch C, Goblirsch S, Brunetti E (2012) Focused assessment with sonography for HIV-associated tuberculosis (FASH) - a short protocol and a pictorial review. Crit Ultrasound J 4:21

5. Heller T, Goblirsch S, Bahlas S, Ahmed M, Giordani MT, Wallrauch C, Brunetti E (2013) Diagnostic value of FASH ultrasound and chest X-ray in HIV co-infected patients with abdominal TB. Int J Tuberc Lung Dis 17:342-344

6. van Hoving DJ, Lamprecht HH, Stander M, Vallabh K, Fredericks D, Louw P, Muller M, Malan JJ (2012) Adequacy of the emergency point-of-care ultrasound core curriculum for the local burden of disease in South Africa. Emerg Med J 30:312-315

7. Heuvelings CC, Bélard S, Janssen S, Wallrauch C, Grobusch MP, Brunetti E, Giordani MT, Heller T (2016) Chest ultrasonography in patients with HIV: a case series and review of the literature. Infection 44:1-10 
8. Hunter L, Bélard S, Janssen S, van Hoving DJ, Heller T (2016) Miliary tuberculosis: sonographic pattern in chest ultrasound. Infection 44:243-246

9. Kaplan JE, Hanson D, Dworkin MS, FrederikT, Bertolli J, Lindegren ML, Holmberg S, Jones JL (2000) Epidemiology of human immunodeficiency virus-associated opportunistic infections in the United States in the era of highly active antiretroviral therapy. Clin Infect Dis 30(Suppl 1):S5-S14

10. Sepkowitz KA (2002) Opportunistic infections in patients with and patients without acquired immunodeficiency syndrome. Clin Infect Dis 34:1293

11. Wang HW, Lin CC, Kuo CF, Lee CM (2011) Mortality predictors of Pneumocystis jirovecii pneumonia in human immunodeficiency virus-infected patients at presentation: experience in a tertiary care hospital of northern Taiwan. J Microbiol Immunol Infect 44:274-281

12. Palella FJ Jr, Delaney KM, Moorman AC, Loveless MO, Fuhrer J, Satten GA, Aschman D, Holmberg SD (1998) Declining morbidity and mortality among patients with advanced human immunodeficiency virus infection. HIV Outpatient Study Investigators. N Engl J Med 338:853-860

13. Lichtenberger JP 3rd, Sharma A, Zachary KC, Krishnam MS, Greene RE, Shepard JA, Wu CC (2012) What a differential a virus makes: a practical approach to thoracic imaging findings in the context of HIV infectionpart 1, pulmonary findings. Am J Roentgenol 198:1295-1304

14. Chou CW, Chao HS, Lin FC, Tsai HC, Yuan WH, Chang SC (2015) Clinical usefulness of HRCT in assessing the severity of Pneumocystis jirovecii pneumonia: a cross-sectional study. Medicine (Baltimore) 94:e768
15. Walzer PD, Eans HE, Copas AJ, Edwards SG, Grant AD, Miller RF (2008) Early predictors of mortality from Pneumocystis jirovecii pneumonia in HIV-infected patients: 1985-2006. Clin Infect Dis 46:625-633

16. Brunetti E, Heller T, Richter J, Kaminstein D, Youkee D, Giordani MT, Goblirsch S, Tamarozzi F (2016) Application of ultrasonography in the diagnosis of infectious diseases in resource-limited settings. Curr Infect Dis Rep 18:6

17. Bélard S, Tamarozzi F, Bustinduy AL, Wallrauch C, Grobusch MP, Kuhn W, Brunetti E, Joekes E, Heller T (2016) Point-of-care ultrasound assessment of tropical infectious diseases - a review of applications and perspectives. Am J Trop Med Hyg 94:8-21

18. Volpicelli G, Elbarbary M, Blaivas M, Lichtenstein DA, Mathis G, Kirkpatrick AW, Melniker L, Gargani L, Noble VE, Via G, Dean A, Tsung JW, Soldati G, Copetti R, Bouhemad B, Reissig A, Agricola E, Rouby JJ, Albelot C, Liteplo A, Sargsyan A, Silva F, Hoppmann R, Breitkreutz R, Seibel A, Neri L, Storti E, Petrovic T, International Liaison Commtee on Lung Ultrasound (ILC-LUS) for International Consensus Conference on Lung Ultrasound (ICC-LUS) (2012) International evidence-based recommendations for point-of-care lung ultrasound. Intensive Care Med 38:577-591

19. Settnes OP, Genner J (1986) Pneumocystis carinii in human lungs at autopsy. Scand J Infect Dis 18:489-496

\section{Submit your manuscript to a SpringerOpen ${ }^{\odot}$ journal and benefit from:}

- Convenient online submission

- Rigorous peer review

- Open access: articles freely available online

- High visibility within the field

- Retaining the copyright to your article

Submit your next manuscript at $\boldsymbol{\nabla}$ springeropen.com 